\title{
MULTI-LINEAR ISOMETRIES ON SPACES OF VECTOR-VALUED CONTINUOUS FUNCTIONS
}

\author{
MALIHEH HOSSEINI AND JUAN J. FONT
}

\begin{abstract}
In this paper we study multilinear isometries defined on certain subspaces of vectorvalued continuous functions. We provide conditions under which such maps can be properly represented. Our results contain all known results concerning linear and bilinear isometries defined between spaces of continuous functions. The key result is a vector-valued version of the additive Bishop's Lemma, which we think that has interest in itself.
\end{abstract}

\section{INTRODUCTION}

Throughout this paper, for a compact Hausdorff space $X$ and a normed space $E$, we denote the space of all $E$-valued continuous functions on $X$ by $C(X, E)$. We set $C(X)$ if $E$ is the scalar field.

Let $A_{1}, \ldots, A_{k}$ be subspaces of continuous functions on compact Hausdorff spaces $X_{1}, \ldots, X_{k}$, respectively, and let $Z$ be a compact Hausdorff space. A $k$-linear map $T: A_{1} \times \ldots \times A_{k} \longrightarrow C(Z)$ is called a multilinear (or k-linear) isometry if

$$
\left\|T\left(f_{1}, \ldots, f_{k}\right)\right\|_{\infty}=\prod_{i=1}^{k}\left\|f_{i}\right\|_{\infty} \quad\left(\left(f_{1}, \ldots, f_{k}\right) \in A_{1} \times \ldots \times A_{k}\right),
$$

where $\|\cdot\|_{\infty}$ denotes the supremum norm.

An important generalization of the famous Banach-Stone theorem, which characterizes surjective linear isometries of the space $C(X)$, was given by Holsztyński in [6] (see also [1]), where he studied non-surjective linear isometries from $C(X)$ to $C(Y)$, that is, 1-linear isometries. More recently, in [9], Moreno and Rodriguez proved the following bilinear (or 2-linear) version of Holsztyński's theorem:

Let $T: C(X) \times C(Y) \longrightarrow C(Z)$ be a bilinear isometry. Then there exist a closed subset $Z_{0}$ of $Z$, a surjective continuous mapping $h: Z_{0} \longrightarrow X \times Y$ and a norm-one continuous function $a \in C(Z)$ such that $T(f, g)(z)=a(z) f\left(\pi_{X}(h(z))\right) g\left(\pi_{Y}(h(z))\right)$ for all $z \in Z_{0}$ and every pair $(f, g) \in C(X) \times C(Y)$,

Key words and phrases: multi-linear isometry, bilinear isometry, additive Bishop's Lemma, spaces of vector-valued continuous functions.

2010 Mathematics Subject Classification. Primary 47B38, 46E40; Secondary 47B33.

Research of J.J. Font was partially supported by Universitat Jaume I (Projecte P11B2014-35), Generalitat Valenciana (Projecte AICO/2016/030) and Spanish government (MTM2016-77143-P).

This work was partially supported by a grant from the Simons Foundation. 
where $\pi_{X}$ and $\pi_{Y}$ are projection maps. The proof of this result relies heavily on the powerful Stone-Weierstrass theorem.

In [7] (see also [4]), based on a new version of the additive Bishop's Lemma, the authors extended the above results to multilinear isometries of function algebras on locally compact Hausdorff spaces, a context where the Stone-Weierstrass theorem is not applicable.

On the other hand, the concept of multilinear isometry can be naturally extended to the context of spaces of vector-valued continuous functions. In this context, Jerison [8] investigated a formulation of the Banach-Stone theorem for $C(X, E)$-spaces, where $E$ is a strictly convex Banach space. In 1978, a vector analogue of Holsztyński's theorem was obtained by Cambern ([3]). Namely, he considered 1-linear isometries from $C(X, E)$ into $C(Y, F)$ assuming that $E$ and $F$ are normed spaces and $F$ is strictly convex. In this vector-valued context, examples of bilinear isometries can be found, for instance, in [10, Proposition 5.2], where the author provided certain compact spaces $X$ and Banach spaces $E$ for which there exists a bilinear isometry $T: C(X, E) \times C(X, E) \longrightarrow C(Y, E)$. In [5], the authors obtained conditions under which a representation of such bilinear isometries on this setting can be obtained, which is to say, a vector-valued version of the results in [9]. They proved that, if $F$ is a strictly convex Banach space and if $T: C\left(X, E_{1}\right) \times C\left(Y, E_{2}\right) \longrightarrow C(Z, F)$ is a bilinear isometry which is stable on constants (see Remark 4.3 for its definition), then there exists a nonempty subset $Z_{0}$ of $Z$, a surjective continuous mapping $h: Z_{0} \longrightarrow X \times Y$ and a continuous function $\omega: Z_{0} \longrightarrow \operatorname{Bil}\left(E_{1} \times E_{2}, F\right)$ such that

$$
T(f, g)(z)=\omega(z)\left(f\left(\pi_{X}(h(z))\right), g\left(\pi_{Y}(h(z))\right)\right)
$$

for all $z \in Z_{0}$ and every pair $(f, g) \in C\left(X, E_{1}\right) \times C\left(Y, E_{2}\right)$, where $\operatorname{Bil}\left(E_{1} \times E_{2}, F\right)$ denotes the space of jointly continuous bilinear maps from $E_{1} \times E_{2}$ into $F$ equipped with the strong operator topology.

In this paper we focus on multilinear isometries defined on certain subspaces of vector-valued continuous functions. We provide a new weaker condition than the stability on constants considered in [5], which allows us to obtain a complete representation of such isometries. In particular, we describe multilinear isometries of $C(X, E)$-spaces for normed spaces $E$ which are not necessarily Banach spaces (compare with [5]). Our results include, basically, all known results concerning linear and bilinear isometries defined between spaces of (both scalar-valued and vector-valued) continuous functions. The key result is a vector-valued version of the additive Bishop's Lemma, which we think that has interest in itself. 


\section{PRELIMINARIES}

Let $X$ be a compact Hausdorff space and $E$ be a normed space. For each $e \in E$, let $\widehat{e}$ denote the function in $C(X, E)$ which is constantly $e$ on $X$. Moreover, for any $f \in C(X, E)$, set $M_{f}:=\{x \in$ $\left.X:\|f(x)\|=\|f\|_{\infty}\right\}$ which is a nonempty compact subset of $X$.

For any normed space $E, S_{E}$ denotes the unit sphere of $E$. A normed space $E$ is called strictly convex if each $e \in S_{E}$ is an extreme point of the closed unit ball of $E$. Especially, for each $e_{1}, e_{2} \in$ $E \backslash\{0\}$, we have $\left\|e_{1}\right\|,\left\|e_{2}\right\|<\max \left\{\left\|e_{1}+e_{2}\right\|,\left\|e_{1}-e_{2}\right\|\right\}$.

Let $X$ be a compact Hausdorff space and $E$ be a Banach space. We say a subspace $A(X, E)$ of $C(X, E)$ is $\star$-regular if for each $e \in S_{E}, x \in X$ and neighborhood $V$ of $x$, there exists a function $f \in A(X, E)$ such that $f(x)=e,\|f\|_{\infty}=1$ and $f=0$ on $X \backslash V$. Note that, among others, the space of vector-valued continuous functions, (little) Lipschitz functions, n-times continuously differentiable functions ( $n \in \mathbb{N} \cup\{\infty\}$ ), continuous functions of bounded variation and absolutely continuous functions on appropriate compact spaces $\mathrm{X}$, are $\star$-regular.

A normed space $E$ is called strictly convex if each $e \in S_{E}$ is an extreme point of the closed unit ball of $E$. Especially, for each $e_{1}, e_{2} \in E \backslash\{0\}$, we have $\left\|e_{1}\right\|,\left\|e_{2}\right\|<\max \left\{\left\|e_{1}+e_{2}\right\|,\left\|e_{1}-e_{2}\right\|\right\}$.

In the sequel, unless otherwise stated, we will assume that $X_{1}, \ldots, X_{k}, Y$ are compact Hausdorff spaces, $E_{1}, \ldots, E_{k}$ are Banach spaces, and $F$ is a strictly convex Banach space. Furthermore, by $\operatorname{Mul}\left(E_{1} \times \ldots \times E_{k}, F\right)$ we mean the space of jointly continuous multilinear maps from $E_{1} \times \ldots \times E_{k}$ to $F$, endowed with the strong operator topology (SOT). For each $i \in\{1, \ldots, k\}$, let $A\left(X_{i}, E_{i}\right)$ be a

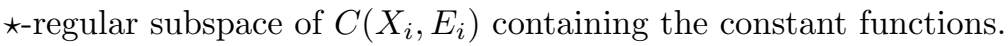

Our aim in this paper is to study multilinear isometries $T: A\left(X_{1}, E_{1}\right) \times \ldots \times A\left(X_{k}, E_{k}\right) \longrightarrow$ $C(Y, F)$. We note that $T$ can be extended naturally to $T: \overline{A\left(X_{1}, E_{1}\right)} \times \ldots \times \overline{A\left(X_{k}, E_{k}\right)} \longrightarrow C(Y, F)$, where for each $i \in\{1, \ldots, k\}, \overline{A\left(X_{i}, E_{i}\right)}$ is the uniform closure of $A\left(X_{i}, E_{i}\right)$ in $C\left(X_{i}, E_{i}\right)$. So we assume, without loss of generality, that each $A\left(X_{i}, E_{i}\right)(i=1, \ldots, k)$ is uniformly closed.

For any $\left(x_{1}, \ldots, x_{k}\right) \in X_{1} \times \ldots \times X_{k}$ and $\left(e_{1}, \ldots, e_{k}\right) \in S_{E_{1}} \times \ldots \times S_{E_{k}}$, we set

$$
C_{x_{i}}^{e_{i}}:=\left\{f \in A\left(X_{i}, E_{i}\right):\|f\|_{\infty}=1, f\left(x_{i}\right)=e_{i}\right\} \quad(i \in\{1, \ldots, k\}) .
$$

Moreover, we define

$$
\mathcal{I}_{x_{1}, \ldots, x_{k}}^{e_{1}, \ldots, e_{k}}:=\left\{y \in Y: y \in M_{T\left(f_{1}, \ldots, f_{k}\right)} \text { for all }\left(f_{1}, \ldots, f_{k}\right) \in C_{x_{1}}^{e_{1}} \times \ldots \times C_{x_{k}}^{e_{k}}\right\}
$$

\section{Required Lemmas}

We deduce our main result (Theorem 4.1) through several lemmas. The first key lemma is an additive version of Bishop's Lemma adapted to the context of spaces of vector-valued continuous functions. 
Lemma 3.1. Assume that $A(X, E)$ is a $\star$-regular closed subspace of $C(X, E), e \in S_{E}$ and $x_{0} \in X$. If $f \in A(X, E)$ with $f\left(x_{0}\right)=0$, then there exists $h \in C_{x_{0}}^{e}$ such that \|\|$f\|+2\| f\left\|_{\infty}\right\| h\|\|_{\infty}=2\|f\|_{\infty}$. In particular, $\|f \pm 2\| f\left\|_{\infty} h\right\|_{\infty}=2\|f\|_{\infty}$.

Proof. We apply an argument similar to the proof of [11, Lemma 1]. For each $n \in \mathbb{N}$, put

$$
V_{n}:=\left\{x \in X:\|f(x)\|<\frac{\|f\|_{\infty}}{2^{n+1}}\right\} .
$$

Clearly, $V_{n}$ is a neighborhood of $x_{0}$ and $V_{n+1} \subseteq V_{n}$ for all $n \in \mathbb{N}$. Since $A(X, E)$ is a $\star$-regular, we can choose $h_{n} \in A(X, E)$ such that $h_{n}\left(x_{0}\right)=e,\left\|h_{n}\right\|_{\infty}=1$ and $h_{n}=0$ on $X \backslash V_{n}$. Define $h=\sum_{n=1}^{\infty} \frac{h_{n}}{2^{n}}$. Obviously, $h \in A(X, E), h\left(x_{0}\right)=e$ and $\|h\|_{\infty}=1$. We now show that \|\|$f\|+2\| f\left\|_{\infty}\right\| h\|\|_{\infty}=2\|f\|_{\infty}$. If $x \in \bigcap_{n=1}^{\infty} V_{n}$, then it is apparent that $f(x)=0$ and so $\|f(x)\|+2\|f\|_{\infty}\|h(x)\| \leq 2\|f\|_{\infty}$. If $x \notin \bigcup_{n=1}^{\infty} V_{n}$, then $h(x)=0$ and so $\|f(x)\|+2\|f\|_{\infty}\|h(x)\| \leq 2\|f\|_{\infty}$. Finally, if $x$ belongs to $V_{1}, \ldots, V_{n-1}$ but not to $V_{n}$, then we have

$$
\|f(x)\|+2\|f\|_{\infty}\|h(x)\|<\frac{\|f\|_{\infty}}{2^{n}}+2\|f\|_{\infty}\left(\sum_{i=1}^{n-1} \frac{1}{2^{i}}\right)<2\|f\|_{\infty} .
$$

Then, from the above arguments and since $\left(\|f\|+2\|f\|_{\infty}\|h\|\right)\left(x_{0}\right)=2\|f\|_{\infty}$, we conclude that \|\|$f\|+2\| f\left\|_{\infty}\right\| h\|\|_{\infty}=2\|f\|_{\infty}$. In particular, it is evident that $\|f \pm 2\| f\left\|_{\infty} h\right\|_{\infty}=2\|f\|_{\infty}$.

Lemma 3.2. For any $\left(x_{1}, \ldots, x_{k}\right) \in X_{1} \times \ldots \times X_{k}$ and $\left(e_{1}, \ldots, e_{k}\right) \in S_{E_{1}} \times \ldots \times S_{E_{k}}$, the set $\mathcal{I}_{x_{1}, \ldots, x_{k}}^{e_{1}, \ldots, e_{k}}$ is nonempty.

Proof. Since for each $\left(f_{1}, \ldots, f_{k}\right) \in C_{x_{1}}^{e_{1}} \times \ldots \times C_{x_{k}}^{e_{k}}$, the set $M_{T\left(f_{1}, \ldots, f_{k}\right)}$ is a compact subset of $Y$, then it is enough to show that the family $\left\{M_{T\left(f_{1}, \ldots, f_{k}\right)}:\left(f_{1}, \ldots, f_{k}\right) \in C_{x_{1}}^{e_{1}} \times \ldots \times C_{x_{k}}^{e_{k}}\right\}$ has the finite intersection property. To see, assume that $\left(f_{1}^{1}, \ldots, f_{k}^{1}\right), \ldots,\left(f_{1}^{n}, \ldots, f_{k}^{n}\right)$ belong to $C_{x_{1}}^{e_{1}} \times \ldots \times C_{x_{k}}^{e_{k}}$. Define

$$
f_{i}:=\frac{1}{n} \sum_{j=1}^{n} f_{i}^{j}, \quad i \in\{1, \ldots, k\}
$$

It is clear that $\left(f_{1}, \ldots, f_{k}\right) \in C_{x_{1}}^{e_{1}} \times \ldots \times C_{x_{k}}^{e_{k}}$, whence $\left\|T\left(f_{1}, \ldots, f_{k}\right)\right\|_{\infty}=\left\|f_{1}\right\|_{\infty} \ldots\left\|f_{k}\right\|_{\infty}=1$. Then there is a point $y_{0} \in Y$ such that

$$
1=\left\|T\left(f_{1}, \ldots, f_{k}\right)\left(y_{0}\right)\right\|=\frac{1}{n^{k}}\left\|\sum_{1 \leq i_{1}, \ldots, i_{k} \leq n} T\left(f_{1}^{i_{1}}, \ldots, f_{k}^{i_{k}}\right)\left(y_{0}\right)\right\| .
$$

Since for each $1 \leq i_{1}, \ldots, i_{k} \leq n,\left(f_{1}^{i_{1}}, \ldots, f_{k}^{i_{k}}\right) \in C_{x_{1}}^{e_{1}} \times \ldots \times C_{x_{k}}^{e_{k}},\left\|T\left(f_{1}^{i_{1}}, \ldots, f_{k}^{i_{k}}\right)\right\|_{\infty}=1$, and so we conclude that $\left\|T\left(f_{1}^{i_{1}}, \ldots, f_{k}^{i_{k}}\right)\left(y_{0}\right)\right\|=1$. In particular, $y_{0} \in \bigcap_{i=1}^{n} M_{T\left(f_{1}^{i}, \ldots, f_{k}^{i}\right)}$. Therefore $\bigcap_{i=1}^{n} M_{T\left(f_{1}^{i}, \ldots, f_{k}^{i}\right)} \neq \emptyset$, as desired. 
Lemma 3.3. Let $\left(x_{1}, \ldots, x_{k}\right) \in X_{1} \times \ldots \times X_{k},\left(e_{1}, \ldots, e_{k}\right) \in S_{E_{1}} \times \ldots \times S_{E_{k}}$, let also $I, J$ be two disjoint sets with $I \neq \emptyset$ and $I \cup J=\{1, \ldots, k\}$. If for each $j \in J, h_{j} \in C_{x_{j}}^{e_{j}}$ and for each $i \in I$, $f_{i} \in A\left(X_{i}, E_{i}\right)$ with $f_{i}\left(x_{i}\right)=0$, then $T\left(F_{1}, \ldots, F_{k}\right)(y)=0$ for all $y \in \mathcal{I}_{x_{1}, \ldots, x_{k}}^{e_{1}, \ldots, e_{k}}$, where $F_{t}=f_{t}$ if $t \in I$ and $F_{t}=h_{t}$ if $t \in J$.

Proof. Contrary to what we claim, suppose that there exists $y_{0} \in \mathcal{I}_{x_{1}, \ldots, x_{k}}^{e_{1}, \ldots, e_{k}}$ such that $T\left(F_{1}, \ldots, F_{k}\right)\left(y_{0}\right)=$ $e \neq 0$. For each $i \in I$, by Lemma 3.1, there exists a function $h_{i} \in C_{x_{i}}^{e_{i}}$ such that \|\|$f_{i}\left\|+r_{i}\right\| h_{i}\|\|_{\infty}=$ $\left\|f_{i} \pm r_{i} h_{i}\right\|_{\infty}=r_{i}$, where $r_{i}=2\left\|f_{i}\right\|_{\infty}$. Moreover, $T\left(h_{1}, \ldots, h_{k}\right)\left(y_{0}\right)=e_{0} \in S_{F}$ since $\left(h_{1}, \ldots, h_{k}\right) \in$ $C_{x_{1}}^{e_{1}} \times \ldots \times C_{x_{k}}^{e_{k}}$.

Let us suppose that $I=\{1\}$. Then taking into account that $F$ is strictly convex, we have

$$
\begin{aligned}
r_{1} & =\left\|f_{1} \pm r_{1} h_{1}\right\|_{\infty}\left\|h_{2}\right\|_{\infty} \ldots\left\|h_{k}\right\|_{\infty}=\left\|T\left(f_{1} \pm r_{1} h_{1}, h_{2}, \ldots, h_{k}\right)\right\|_{\infty} \\
& \geq\left\|T\left(f_{1}, h_{2}, \ldots, h_{k}\right)\left(y_{0}\right) \pm r_{1} T\left(h_{1}, \ldots, h_{k}\right)\left(y_{0}\right)\right\| \\
& =\left\|e \pm r_{1} e_{0}\right\|>\left\|r_{1} e_{0}\right\|=r_{1},
\end{aligned}
$$

and it is a contradiction showing that $T\left(F_{1}, \ldots, F_{k}\right)(y)=0$ for all $y \in \mathcal{I}_{x_{1}, \ldots, x_{k}}^{e_{1}, \ldots, e_{k}}$. A similar method implies that the result holds for all cases where $\operatorname{card}(I)=1$.

Next suppose that the result is true when $\operatorname{card}(I)=l-1$ and $2 \leq l \leq k$ and we show that the result is held if $\operatorname{card}(I)=l$. Let us first assume that $l<k$. Without loss of generality, suppose that $I=\left\{x_{1}, \ldots, x_{l}\right\}$. Similar to the above argument, from strict convexity of $F$ it follows that

$$
\begin{aligned}
r_{1} r_{2} \ldots r_{l} & =\left\|f_{1} \pm r_{1} h_{1}\right\|_{\infty}\left\|f_{2}+r_{2} h_{2}\right\|_{\infty} \ldots\left\|f_{l}+r_{l} h_{l}\right\|_{\infty}\left\|h_{l+1}\right\|_{\infty} \ldots\left\|h_{k}\right\|_{\infty} \\
& =\left\|T\left(f_{1} \pm r_{1} h_{1}, f_{2}+r_{2} h_{2}, \ldots, f_{l}+r_{l} h_{l}, h_{l+1}, \ldots, h_{k}\right)\right\|_{\infty} \\
& \geq\left\|T\left(f_{1} \pm r_{1} h_{1}, f_{2}+r_{2} h_{2}, \ldots, f_{l}+r_{l} h_{l}, h_{l+1}, \ldots, h_{k}\right)\left(y_{0}\right)\right\| \\
& =\left\|T\left(f_{1}, \ldots, f_{l}, h_{l+1}, \ldots, h_{k}\right)\left(y_{0}\right) \pm r_{1} r_{2} \ldots r_{l} T\left(h_{1}, \ldots, h_{k}\right)\left(y_{0}\right)\right\| \\
& =\left\|e \pm r_{1} \ldots r_{l} e_{0}\right\|>r_{1} \ldots r_{l},
\end{aligned}
$$

which is impossible. This argument shows the validity of the result for the case where $\operatorname{card}(I)<k$.

Now assume that $I=\left\{x_{1}, \ldots, x_{k}\right\}$. We have

$$
\begin{aligned}
r_{1} \ldots r_{k} & =\left\|f_{1} \pm r_{1} h_{1}\right\|_{\infty}\left\|f_{2}+r_{2} h_{2}\right\|_{\infty} \ldots\left\|f_{k}+r_{k} h_{k}\right\|_{\infty} \\
& =\left\|T\left(f_{1} \pm r_{1} h_{1}, f_{2}+r_{2} h_{2}, \ldots, f_{k}+r_{k} h_{k}\right)\right\|_{\infty} \\
& \geq\left\|T\left(f_{1}, \ldots, f_{k}\right)\left(y_{0}\right) \pm r_{1} \ldots r_{k} T\left(h_{1}, \ldots, h_{k}\right)\left(y_{0}\right)\right\| \\
& =\left\|e \pm r_{1} \ldots r_{k} e_{0}\right\|>r_{1} \ldots r_{k},
\end{aligned}
$$

which is again a contradiction showing that $T\left(f_{1}, \ldots, f_{k}\right)(y)=0$ for all $y \in \mathcal{I}_{x_{1}, \ldots, x_{k}}^{e_{1}, \ldots, e_{k}}$. 
Lemma 3.4. Let $\left(x_{1}, \ldots, x_{k}\right)$ and $\left(x_{1}^{\prime}, \ldots, x_{k}^{\prime}\right)$ be distinct elements in $X_{1} \times \ldots \times X_{k}$, and $\left(e_{1}, \ldots, e_{k}\right) \in$ $S_{E_{1}} \times \ldots \times S_{E_{k}}$, then $\mathcal{I}_{x_{1}, \ldots, x_{k}}^{e_{1}, \ldots, e_{k}} \cap \mathcal{I}_{x_{1}^{\prime}, \ldots, x_{k}^{\prime}}^{e_{1}, \ldots, e_{k}}=\emptyset$.

Proof. Contrary to what we claim, suppose that there exists a point $y_{0} \in \mathcal{I}_{x_{1}, \ldots, x_{k}}^{e_{1}, \ldots, e_{k}} \bigcap \mathcal{I}_{x_{1}^{\prime}, \ldots, x_{k}^{\prime}}^{e_{1}, \ldots, e_{k}}$. Since $\left(x_{1}, \ldots, x_{k}\right)$ and $\left(x_{1}^{\prime}, \ldots, x_{k}^{\prime}\right)$ are distinct, the set $L=\left\{i: 1 \leq i \leq k, x_{i} \neq x_{i}^{\prime}\right\}$ is nonempty. For each $i \in L$, we can choose a function $f_{i} \in C_{x_{i}}^{e_{i}}$ such that $f_{i}\left(x_{i}^{\prime}\right)=0$ because $A\left(X_{i}, E_{i}\right)$ is $\star$-regular. Moreover, for each $j \in\{1, \ldots, k\} \backslash L$, we take a function $f_{j}$ in $C_{x_{j}}^{e_{j}}$. Now according to Lemma $3.3, T\left(f_{1}, \ldots, f_{k}\right)\left(y_{0}\right)=0$ since $y_{0} \in \mathcal{I}_{x_{1}^{\prime}, \ldots, x_{k}^{\prime}}^{e_{1}, \ldots, e_{k}}$. On the other hand, $\left\|T\left(f_{1}, \ldots, f_{k}\right)\left(y_{0}\right)\right\|=1$ because $y_{0} \in \mathcal{I}_{x_{1}, \ldots, x_{k}}^{e_{1}, \ldots, e_{k}}$. But it is impossible and so $\mathcal{I}_{x_{1}, \ldots, x_{k}}^{e_{1}, \ldots, e_{k}} \cap \mathcal{I}_{x_{1}^{\prime}, \ldots, x_{k}^{\prime}}^{e_{1}, \ldots, e_{k}}=\emptyset$.

Definition 3.5. For any $\left(x_{1}, \ldots, x_{k}\right) \in X_{1} \times \ldots \times X_{k}$, let

$$
\mathcal{I}_{x_{1}, \ldots, x_{k}}:=\bigcap_{\left(e_{1}, \ldots, e_{k}\right) \in S_{E_{1}} \times \ldots \times S_{E_{k}}} \mathcal{I}_{x_{1}, \ldots, x_{k}}^{e_{1}, \ldots, e_{k}}
$$

It should be noted that according to the above lemma, for any distinct elements $\left(x_{1}, \ldots, x_{k}\right)$ and $\left(x_{1}^{\prime}, \ldots, x_{k}^{\prime}\right)$ in $X_{1} \times \ldots \times X_{k}, \mathcal{I}_{x_{1}, \ldots, x_{k}} \cap \mathcal{I}_{x_{1}^{\prime}, \ldots, x_{k}^{\prime}}=\emptyset$.

Although for each $\left(x_{1}, \ldots, x_{k}\right) \in X_{1} \times \ldots \times X_{k}$ and $\left(e_{1}, \ldots, e_{k}\right) \in S_{E_{1}} \times \ldots \times S_{E_{k}}, \mathcal{I}_{x_{1}, \ldots, x_{k}}^{e_{1}, \ldots, e_{k}}$ is nonempty (Lemma 3.2), we do not know if the set $\mathcal{I}_{x_{1}, \ldots, x_{k}}$ is also non-empty. So we need to introduce an additional property as follows:

Definition 3.6. We say that a k-linear isometry $T$ satisfies $N$-property if for each $\left(x_{1}, \ldots, x_{k}\right) \in$ $X_{1} \times \ldots \times X_{k}, \mathcal{I}_{x_{1}, \ldots, x_{k}} \neq \emptyset$ or, equivalently, there exists $y \in Y$ such that $\left\|T\left(f_{1}, \ldots, f_{k}\right)(y)\right\|=1$ for all functions $\left(f_{1}, \ldots, f_{k}\right)$ with $f_{i}\left(x_{i}\right) \in S_{E_{i}}$ and $\left\|f_{i}\right\|_{\infty}=1$.

Proposition 3.7. Assume that for each $\left(f_{1}, \ldots, f_{k}\right) \in A\left(X_{1}, E_{1}\right) \times \ldots \times A\left(X_{k}, E_{k}\right)$ and $e_{i}, e_{i}^{\prime} \in$ $S_{E_{i}}, i=1, \ldots, k$, we have

$$
M_{T\left(f_{1}, \ldots, f_{i-1}, e_{i}, f_{i+1}, \ldots, f_{k}\right)}=M_{T\left(f_{1}, \ldots, f_{i-1}, e_{i}^{\prime}, f_{i+1}, \ldots, f_{k}\right)} .
$$

Then

$$
\mathcal{I}_{x_{1}, \ldots, x_{k}}^{e_{1}, \ldots, e_{k}}=\mathcal{I}_{x_{1}, \ldots, x_{k}}^{e_{1}^{\prime}, \ldots, e_{k}^{\prime}}
$$

for all $\left(e_{1}, \ldots, e_{k}\right),\left(e_{1}^{\prime}, \ldots, e_{k}^{\prime}\right) \in S_{E_{1}} \times \ldots \times S_{E_{k}}$ and each $\left(x_{1}, \ldots, x_{k}\right) \in X_{1} \times \ldots \times X_{k}$.

Proof. Fix $\left(x_{1}, \ldots, x_{k}\right) \in X_{1} \times \ldots \times X_{k}$ and let $y \in \mathcal{I}_{x_{1}, \ldots, x_{k}}^{e_{1}, \ldots, e_{k}}$, which exists by Lemma $3.2,\left(g_{1}, \ldots, g_{k}\right) \in$ $C_{x_{1}}^{e_{1}} \times \ldots \times C_{x_{k}}^{e_{k}}$ and $\left(f_{1}, \ldots, f_{k}\right) \in C_{x_{1}}^{e_{1}^{\prime}} \times \ldots \times C_{x_{k}}^{e_{k}^{\prime}}$. From Lemma 3.3 , we have $T\left(f_{1}-e_{1}^{\prime}, g_{2}, \ldots, g_{k}\right)(y)=$ 0 and so $T\left(f_{1}, g_{2}, \ldots, g_{k}\right)(y)=T\left(e_{1}^{\prime}, g_{2}, \ldots, g_{k}\right)(y)$. Since $M_{T\left(e_{1}^{\prime}, g_{2}, \ldots, g_{k}\right)}=M_{T\left(e_{1}, g_{2}, \ldots, g_{k}\right)}$ and $y \in$ $M_{T\left(e_{1}, g_{2}, \ldots, g_{k}\right)}$, then we infer that $y \in M_{T\left(f_{1}, g_{2}, \ldots, g_{k}\right)}$. This argument shows that $y \in \mathcal{I}_{x_{1}, x_{2}, \ldots, x_{k}}^{e_{1}^{\prime}, e_{2}, \ldots, e_{k}}$. Thus again by Lemma 3.3 , we have $T\left(f_{1}, f_{2}-e_{2}^{\prime}, g_{3}, \ldots, g_{k}\right)(y)=0$, and so $T\left(f_{1}, f_{2}, g_{3}, \ldots, g_{k}\right)(y)=$ $T\left(f_{1}, e_{2}^{\prime}, g_{3}, \ldots, g_{k}\right)(y)$. Now taking into account that $M_{T\left(f_{1}, e_{2}^{\prime}, g_{3}, \ldots, g_{k}\right)}=M_{T\left(f_{1}, e_{2}, g_{3}, \ldots, g_{k}\right)}$ and $y \in$ 
$\mathcal{I}_{x_{1}, x_{2}, \ldots, x_{k}}^{e_{1}^{\prime}, e_{2}, \ldots, e_{k}}$, it follows that $y \in M_{T\left(f_{1}, f_{2}, g_{3}, \ldots, g_{k}\right)}$. According to this discussion, we deduce that $y \in \mathcal{I}_{x_{1}, x_{2}, x_{3}, \ldots, x_{k}}^{e_{1}^{\prime}, e_{2}^{\prime}, e_{3}, \ldots, e_{k}}$. By continuing this process, finally it is concluded that $y \in \mathcal{I}_{x_{1}, \ldots, x_{k}}^{e_{1}^{\prime}, \ldots, e_{k}^{\prime}}$. Therefore, $\mathcal{I}_{x_{1}, \ldots, x_{k}}^{e_{1}, \ldots, e_{k}} \subseteq \mathcal{I}_{x_{1}, \ldots, x_{k}}^{e_{1}^{\prime}, \ldots, e_{k}^{\prime}}$. Similarly, $\mathcal{I}_{x_{1}, \ldots, x_{k}}^{e_{1}, \ldots, e_{k}} \supseteq \mathcal{I}_{x_{1}, \ldots, x_{k}}^{e_{1}^{\prime}, \ldots, e_{k}^{\prime}}$ and so $\mathcal{I}_{x_{1}, \ldots, x_{k}}^{e_{1}, \ldots, e_{k}}=\mathcal{I}_{x_{1}, \ldots, x_{k}}^{e_{1}^{\prime}, \ldots, e_{k}^{\prime}}$.

It is clear that if $T$ satisfies the assumption in the above proposition, then $T$ satisfies N-property. It should be also noted that N-property is weaker than the condition (stability on constants) proposed in [5] for bilinear isometries (see Remark 4.3).

Lemma 3.8. Let $\left(x_{1}, \ldots, x_{k}\right) \in X_{1} \times \ldots \times X_{k}, I$ and $J$ be two disjoint sets with $I \neq \emptyset$ and $I \cup J=$ $\{1, \ldots, k\}$. If for each $i \in I, f_{i} \in A\left(X_{i}, E_{i}\right)$ with $f_{i}\left(x_{i}\right)=0$, and for each $j \in J, f_{j} \in A\left(X_{j}, E_{j}\right)$, then $T\left(f_{1}, \ldots, f_{k}\right)(y)=0$ for all $y \in \mathcal{I}_{x_{1}, \ldots, x_{k}}$.

Proof. Let $y \in \mathcal{I}_{x_{1}, \ldots, x_{k}}$. We can assume, without loss of generality, that $f_{j}\left(x_{j}\right) \in S_{E_{j}}$ for all $j \in J$. Moreover, for each $i \in I$, let $e_{i}$ be a point in $S_{E_{i}}$. Then $\left(e_{1}, \ldots, e_{k}\right) \in S_{E_{1}} \times \ldots \times S_{E_{k}}$, where $e_{j}=f_{j}\left(x_{j}\right)$ for each $j \in J$, and we choose $\left(h_{1}, \ldots, h_{k}\right) \in C_{x_{1}}^{e_{1}} \times \ldots \times C_{x_{k}}^{e_{k}}$.

First consider the case where $\operatorname{card}(J)=1$. We suppose, without loss of generality, that $J=\{1\}$. By Lemma 3.3, $T\left(h_{1}, f_{2}, \ldots, f_{k}\right)(y)=0$ and $T\left(h_{1}-f_{1}, f_{2}, \ldots, f_{k}\right)(y)=0$. Then from k-linearity of $T$ it follows that $T\left(f_{1}, \ldots, f_{k}\right)(y)=0$. Now assume that $\operatorname{card}(J)=2$ and $J=\{1,2\}$. Again from Lemma 3.3 we have $T\left(f_{1}-h_{1}, f_{2}-h_{2}, f_{3}, \ldots, f_{k}\right)(y)=0, T\left(f_{1}-h_{1}, h_{2}, f_{3}, \ldots, f_{k}\right)(y)=0$, $T\left(h_{1}, f_{2}-h_{2}, f_{3}, \ldots, f_{k}\right)(y)=0$, and $T\left(h_{1}, h_{2}, f_{3}, \ldots, f_{k}\right)(y)=0$, that specially the latter three equations guarantee that $T\left(f_{1}, h_{2}, f_{3}, \ldots, f_{k}\right)(y)=0$ and $T\left(h_{1}, f_{2}, \ldots, f_{k}\right)(y)=0$. Now, k-linearity of $T$ easily yields that $T\left(f_{1}, \ldots, f_{k}\right)(y)=0$. A similar discussion holds for all cases where $\operatorname{card}(J)=2$.

By continuing this progress we conclude that the result is also true for all cases where $\operatorname{card}(J)>$ 2 .

Lemma 3.9. If $\left(x_{1}, \ldots, x_{k}\right) \in X_{1} \times \ldots \times X_{k},\left(f_{1}, \ldots, f_{k}\right) \in A\left(X_{1}, E_{1}\right) \times \ldots \times A\left(X_{k}, E_{k}\right)$ and $y \in \mathcal{I}_{x_{1}, \ldots, x_{k}}$, then $T\left(f_{1}, \ldots, f_{k}\right)(y)=T\left(\widehat{f_{1}\left(x_{1}\right)}, \ldots, \widehat{f_{k}\left(x_{k}\right)}\right)(y)$.

Proof. From the previous lemma, we have $T\left(f_{1}-\widehat{f_{1}\left(x_{1}\right)}, f_{2}, \ldots, f_{k}\right)(y)=0$, which implies that

$$
T\left(f_{1}, \ldots, f_{k}\right)(y)=T\left(\widehat{f_{1}\left(x_{1}\right)}, f_{2}, \ldots, f_{k}\right)(y)
$$

Again by the previous lemma, $T\left(\widehat{f_{1}\left(x_{1}\right)}, f_{2}-\widehat{f_{2}\left(x_{2}\right)}, f_{3}, \ldots, f_{k}\right)(y)=0$, and so $T\left(\widehat{f_{1}\left(x_{1}\right)}, f_{2}, \ldots, f_{k}\right)(y)=$ $T\left(\widehat{f_{1}\left(x_{1}\right)}, \widehat{f_{2}\left(x_{2}\right)}, f_{3}, \ldots, f_{k}\right)(y)$. Consequently we have proved that

$$
T\left(f_{1}, \ldots, f_{k}\right)(y)=T\left(\widehat{f_{1}\left(x_{1}\right)}, \widehat{f_{2}\left(x_{2}\right)}, f_{3}, \ldots, f_{k}\right)(y) .
$$

By continuing this progress we derive that $T\left(f_{1}, \ldots, f_{k}\right)(y)=T\left(\widehat{f_{1}\left(x_{1}\right)}, \ldots, \widehat{f_{k}\left(x_{k}\right)}\right)(y)$. 


\section{Main Result}

Now we can state our main result.

Theorem 4.1. Let $T: A\left(X_{1}, E_{1}\right) \times \ldots \times A\left(X_{k}, E_{k}\right) \longrightarrow C(Y, F)$ be a k-linear isometry satisfying $N$-property. Then there exist a nonempty subset $Y_{0}$ of $Y$, a continuous surjective map $\varphi: Y_{0} \longrightarrow$ $X_{1} \times \ldots \times X_{k}$, a continuous function $\omega: Y_{0} \longrightarrow \operatorname{Mul}\left(E_{1} \times \ldots \times E_{k}, F\right)$ such that

$$
T\left(f_{1}, \ldots, f_{k}\right)(y)=\omega(y)\left(f_{1}\left(\pi_{1}(\varphi(y))\right), \ldots, f_{k}\left(\pi_{k}(\varphi(y))\right)\right),
$$

for all $\left(f_{1}, \ldots, f_{k}\right) \in A\left(X_{1}, E_{1}\right) \times \ldots \times A\left(X_{k}, E_{k}\right)$ and $y \in Y_{0}$, where $\pi_{i}$ is the ith projection map.

Proof. Set $Y_{0}:=\left\{y \in Y: y \in \mathcal{I}_{x_{1}, \ldots, x_{k}}\right.$ for some $\left.\left(x_{1}, \ldots, x_{k}\right) \in X_{1} \times \ldots \times X_{k}\right\}$, which is a nonempty subset of $Y$ by N-property.

We define the map $\varphi: Y_{0} \longrightarrow X_{1} \times \ldots \times X_{k}$ by $\varphi(y):=\left(x_{1}, \ldots, x_{k}\right)$ if $y \in \mathcal{I}_{x_{1}, \ldots, x_{k}}$. From Lemma 3.4 , it is apparent that $\varphi$ is well-defined. Meantime, since $T$ satisfies N-property, we infer that $\varphi$ is surjective.

Next, define the map $\omega: Y_{0} \longrightarrow \operatorname{Mul}\left(E_{1} \times \ldots \times E_{k}, F\right)$ as $\omega(y)\left(e_{1}, \ldots, e_{k}\right)=T\left(\widehat{e_{1}}, \ldots, \widehat{e_{k}}\right)(y)$ for all $\left(e_{1}, \ldots, e_{k}\right) \in E_{1} \times \ldots \times E_{k}$ and $y \in Y_{0}$. We now prove that $\omega$ is continuous. Let $\left(y_{\alpha}\right)$ be a net in $Y_{0}$ converging to $y \in Y_{0}$. Fix $\left(e_{1}, \ldots, e_{k}\right) \in E_{1} \times \ldots \times E_{k}$. From the definition of $\omega$ we deduce that

$$
\left\|\omega\left(y_{\alpha}\right)\left(e_{1}, \ldots, e_{k}\right)-\omega(y)\left(e_{1}, \ldots, e_{k}\right)\right\|=\left\|T\left(\widehat{e_{1}}, \ldots, \widehat{e_{k}}\right)\left(y_{\alpha}\right)-T\left(\widehat{e_{1}}, \ldots, \widehat{e_{k}}\right)(y)\right\| \rightarrow 0,
$$

because of the continuity of $T\left(\widehat{e_{1}}, \ldots, \widehat{e_{k}}\right)$. Hence $\omega$ is continuous.

Now if $\left(f_{1}, \ldots, f_{k}\right) \in A\left(X_{1}, E_{1}\right) \times \ldots \times A\left(X_{k}, E_{k}\right)$ and $y \in \mathcal{I}_{x_{1}, \ldots, x_{k}}$ for some $\left(x_{1}, \ldots, x_{k}\right) \in X_{1} \times$ $\ldots \times X_{k}$, then by Lemma 3.9 we have

$$
\begin{aligned}
T\left(f_{1}, \ldots, f_{k}\right)(y) & =T\left(\widehat{f_{1}\left(x_{1}\right)}, \ldots, \widehat{f_{k}\left(x_{k}\right)}\right)(y) \\
& =\omega(y)\left(f_{1}\left(x_{1}\right), \ldots, f_{k}\left(x_{k}\right)\right)
\end{aligned}
$$

Indeed, we get

$$
T\left(f_{1}, \ldots, f_{k}\right)(y)=\omega(y)\left(f_{1}\left(\pi_{1}(\varphi(y))\right), \ldots, f_{k}\left(\pi_{k}(\varphi(y))\right)\right),
$$

for all $\left(f_{1}, \ldots, f_{k}\right) \in A\left(X_{1}, E_{1}\right) \times \ldots \times A\left(X_{k}, E_{k}\right)$ and $y \in Y_{0}$.

Finally, we show the continuity of $\varphi$. Assume that $y \in Y_{0}$ and $\varphi(y)=\left(x_{1}, \ldots, x_{k}\right)$. Let $V_{1} \times \ldots \times V_{k}$ be a neighborhood of $\left(x_{1}, \ldots, x_{k}\right)$ in $X_{1} \times \ldots \times X_{k}$. For each $i, i=1, \ldots, k$, choose $f_{i} \in C_{x_{i}}^{e_{i}}$ such that $f_{i}=0$ on $X_{i} \backslash V_{i}$ for some $\left(e_{1}, \ldots, e_{k}\right) \in S_{E_{1}} \times \ldots \times S_{E_{k}}$. Define

$$
W:=\left\{z \in Y_{0}:\left\|T\left(f_{1}, \ldots, f_{k}\right)(z)\right\|>\frac{1}{2}\right\} .
$$


Clearly, $W$ is a neighborhood of $y$ since $\varphi(y)=\left(x_{1}, \ldots, x_{k}\right)$ and so $\left\|T\left(f_{1}, \ldots, f_{k}\right)(y)\right\|=1$. Moreover we claim that $\varphi(W) \subseteq V_{1} \times \ldots \times V_{k}$. To this end, let $z \in W$ and $\varphi(z)=\left(x_{1}^{\prime}, \ldots, x_{k}^{\prime}\right)$. From the representation of $T$, we conclude that

$$
\begin{aligned}
\frac{1}{2} & <\left\|T\left(f_{1}, \ldots, f_{k}\right)(z)\right\|=\left\|\omega(z)\left(f_{1}\left(\pi_{1}(\varphi(z))\right), \ldots, f_{k}\left(\pi_{k}(\varphi(z))\right)\right)\right\| \\
& =\left\|\omega(z)\left(f_{1}\left(x_{1}^{\prime}\right), \ldots, f_{k}\left(x_{k}^{\prime}\right)\right)\right\| \\
& =\left\|T\left(\widehat{f_{1}\left(x_{1}^{\prime}\right)}, \ldots, \widehat{f_{k}\left(x_{k}^{\prime}\right)}\right)(z)\right\| \\
& \leq\left\|T\left(\widehat{f_{1}\left(x_{1}^{\prime}\right)}, \ldots, \widehat{f_{k}\left(x_{k}^{\prime}\right)}\right)\right\|_{\infty} \\
& =\left\|f_{1}\left(x_{1}^{\prime}\right)\right\| \ldots\left\|f_{k}\left(x_{k}^{\prime}\right)\right\|,
\end{aligned}
$$

which especially shows that for any $i \in\{1, \ldots, k\}, f_{i}\left(x_{i}^{\prime}\right) \neq 0$, and consequently, $x_{i}^{\prime} \in V_{i}$. Hence $\left(x_{1}^{\prime}, \ldots, x_{k}^{\prime}\right) \in V_{1} \times \ldots \times V_{k}$, as asserted. Therefore, $\varphi$ is continuous.

The next result describes multilinear isometries of $C(X, E)$-spaces for normed spaces $E$ which are not necessarily Banach spaces.

Corollary 4.2. If $E_{1}, \ldots, E_{k}, F$ are normed spaces and $T: C\left(X_{1}, E_{1}\right) \times \ldots \times C\left(X_{k}, E_{k}\right) \longrightarrow C(Y, F)$ is a $k$-linear isometry satisfying $N$-property, then there exist a nonempty subset $Y_{0}$ of $Y$, a continuous surjective map $\varphi: Y_{0} \longrightarrow X_{1} \times \ldots \times X_{k}$, a continuous function $\omega: Y_{0} \longrightarrow \operatorname{Mul}\left(E_{1} \times \ldots \times E_{k}, F\right)$ such that for each $\left(f_{1}, \ldots, f_{k}\right) \in C\left(X_{1}, E_{1}\right) \times \ldots \times C\left(X_{k}, E_{k}\right)$ and $y \in Y_{0}$,

$$
T\left(f_{1}, \ldots, f_{k}\right)(y)=\omega(y)\left(f_{1}\left(\pi_{1}(\varphi(y))\right), \ldots, f_{k}\left(\pi_{k}(\varphi(y))\right)\right) .
$$

Proof. According to the notations from the proof of Lemma 3.1, we can choose $k_{n} \in C(X)$ such that $k_{n}\left(x_{0}\right)=1=\left\|k_{n}\right\|_{\infty}$ and $k_{n}=0$ on $X \backslash V_{n}$. Now putting $h_{n}=k_{n} e$ and defining $h=\sum_{n=1}^{\infty} \frac{h_{n}}{2^{n}}$, we can obtain Lemma 3.1. Then following the rest of steps, we can obtain the result.

Remark 4.3. (1) The next example shows that our assumption (see Definition 3.6) is weaker than the assumption stability on constants given in [5]. Let us first recall that a bilinear isometry $T$ : $C\left(X_{1}, E_{1}\right) \times C\left(X_{2}, E_{2}\right) \longrightarrow C(Y, F)$ is said to be stable on constants if for each $(f, g) \in C\left(X_{1}, E_{1}\right) \times$ $C\left(X_{2}, E_{2}\right)$ and $y \in Y$ we have

$$
\begin{aligned}
& \left\|T\left(f, \widehat{e_{2}}\right)(y)\right\|=\left\|T\left(f, \widehat{e_{2}^{\prime}}\right)(y)\right\| \quad\left(e_{2}, e_{2}^{\prime} \in S_{E_{2}}\right), \\
& \left\|T\left(\widehat{e_{1}}, g\right)(y)\right\|=\left\|T\left(\widehat{e_{1}^{\prime}}, g\right)(y)\right\| \quad\left(e_{1}, e_{1}^{\prime} \in S_{E_{1}}\right) .
\end{aligned}
$$

In particular, we have $M_{T\left(f, \widehat{e_{2}}\right)}=M_{T\left(f, \widehat{e_{2}^{\prime}}\right)}$ and $M_{T\left(\widehat{e_{1}}, g\right)}=M_{T\left(\widehat{e_{1}^{\prime}}, g\right)}$ for all $(f, g) \in C\left(X_{1}, E_{1}\right) \times$ $C\left(X_{2}, E_{2}\right)$ and $\left(e_{1}, e_{2}\right),\left(e_{1}^{\prime}, e_{2}^{\prime}\right) \in S_{E_{1}} \times S_{E_{2}}$. By Proposition 3.7, we infer that $\mathcal{I}_{x_{1}, x_{2}}=\mathcal{I}_{x_{1}, x_{2}}^{e_{1}, e_{2}}$ for all $\left(e_{1}, e_{2}\right) \in S_{E_{1}} \times S_{E_{2}}$ and $\left(x_{1}, x_{2}\right) \in X_{1} \times X_{2}$. 
Let $X_{1}=\left\{x_{1}\right\}, X_{2}=\left\{x_{2}\right\}$, and $Y=$ the one point compactification $\mathbb{N} \cup\{\infty\}$ of $\mathbb{N}$. Define $T: C\left(X_{1}, c_{0}\right) \times C\left(X_{2}\right) \longrightarrow C\left(Y, c_{0}\right)$ by $T\left(f_{1}, f_{2}\right)(1)=T\left(f_{1}, f_{2}\right)(2)=f_{1}\left(x_{1}\right) f_{2}\left(x_{2}\right), T\left(f_{1}, f_{2}\right)(\infty)=0$, and for each $n \geq 3, T\left(f_{1}, f_{2}\right)(n)=<f_{1}\left(x_{1}\right), e_{n}>f_{2}\left(x_{2}\right) e_{n}$, where $e_{n}=(0, \ldots, 0,1,0, .$.$) , that is, all$ coordinates are zero except for a 1 in the $n$th coordinate, and $<f_{1}\left(x_{1}\right), e_{n}>$ is the $n$th term of $f_{1}\left(x_{1}\right)$. It is apparent that $T$ is a bilinear isometry and we have

$\mathcal{I}_{x_{1}, x_{2}}^{e_{1}, 1}=\{1,2\}, \mathcal{I}_{x_{1}, x_{2}}^{e_{3}, 1}=\{1,2,3\}, \mathcal{I}_{x_{1}, x_{2}}^{e_{4}, 1}=\{1,2,4\}, \mathcal{I}_{x_{1}, x_{2}}^{e_{1}, i}=\{1,2\}, \cdots$

Clearly, $\mathcal{I}_{x_{1}, x_{2}}=\{1,2\}$. Consequently, $T$ satisfies N-property while $T$ is not stable on constants by the previous paragraph.

(2) By considering $k=2$ and $A\left(X_{i}, E_{i}\right)=C\left(X_{i}, E_{i}\right)$ in Corollary 4.2, we obtain the main result in [5] under a weaker condition (as shown in (1)) and a different method for the case where $E_{1}$ and $E_{2}$ are not necessarily Banach spaces.

We note that for the case where $E_{1}=\ldots=E_{k}=\mathbb{C}$ or $\mathbb{R}$, from the $k$-linearity of $T$ it follows easily that for each $\left(x_{1}, \ldots, x_{k}\right) \in X_{1} \times \ldots \times X_{k}$ and $\left(e_{1}, \ldots, e_{k}\right) \in \mathbb{T}^{k}\left(\right.$ or $\left.\{1,-1\}^{k}\right), \mathcal{I}_{x_{1}, \ldots, x_{k}}=\mathcal{I}_{x_{1}, \ldots, x_{k}}^{e_{1}, \ldots, e_{k}}$, which is a non-empty set. The following result, which is an easy consequence of Theorem 4.1, is a generalization of the main theorems in [7] and [9] for certain function spaces.

Corollary 4.4. Suppose that $A_{i}$ is a «-regular subspace of $C\left(X_{i}\right)(i=1, \ldots, k)$ and $T: A_{1} \times \ldots \times$ $A_{k} \longrightarrow C(Y)$ is a k-linear isometry. Then there exist a nonempty subset $Y_{0}$ of $Y$, a continuous surjective map $\varphi: Y_{0} \longrightarrow X_{1} \times \ldots \times X_{k}$, and a unimodular continuous function $\omega: Y_{0} \longrightarrow \mathbb{T}$ such that

$$
T\left(f_{1}, \ldots, f_{k}\right)(y)=\omega(y) \prod_{i=1}^{k} f_{i}\left(\pi_{i}(\varphi(y))\right)
$$

for all $\left(f_{1}, \ldots, f_{k}\right) \in A_{1} \times \ldots \times A_{k}$ and $y \in Y_{0}$, where $\pi_{i}$ is the ith projection map.

Let us consider the case where $k=1$. It is worth pointing out that in this case our assumption can be dropped. Actually, from Lemma 3.3, we obtain this key result: if $x \in X$ and $f(x)=0$, then $T f(y)=0$ for all $y \in \bigcup_{e \in S_{E}} \mathcal{I}_{x}^{e}$, which allows us to define $\omega$ as in the proof of Theorem 4.1.

Corollary 4.5. Let $A(X, E)$ be a -regular subspace of $C(X, E)$ and $T: A(X, E) \longrightarrow C(Y, F)$ be a linear isometry. Then there exist a nonempty subset $Y_{0}$ of $Y$, a continuous surjective map $\varphi: Y_{0} \longrightarrow X$, a continuous function $\omega$ from $Y_{0}$ to the space of continuous linear operators of $E$ into $F$ such that $T(f)(y)=\omega(y)(f(\varphi(y)))$ for all $f \in A(X, E)$ and $y \in Y_{0}$.

We finally remark that when $A(X, E)=C(X, E)$, Corollaries 4.2 and 4.5 reduce to Cambern's result [3] which is the vector-valued analogue of the celebrated Holsztyński's theorem. 


\section{REFERENCES}

[1] J. Araujo and J.J. Font, Linear isometries between subspaces of continuous functions, Trans. Amer. Math. Soc. 349 (1997) 413-428.

[2] A. Browder, Introduction to Function Algebras, W. A. Benjamin, New York-Amsterdam, 1969.

[3] M. Cambern, A Holsztynski theorem for spaces of continuous vector-valued functions, Studia Math. 63 (1978) 213-217.

[4] J.J. Font and M. Sanchis, Bilinear isometries on subspaces of continuous functions, Math. Nachr. 283 (2010) 568-572.

[5] J.J. Font and M. Sanchis, Bilinear isometries on spaces of vector-valued continuous functions, J. Math. Anal. Appl. 385 (2012) 340-344.

[6] W. Holsztyński, Continuous mappings induced by isometries of spaces of continuous functions, Studia Math. 26 (1966) 133-136.

[7] M. Hosseini, J.J. Font and M. Sanchis, Multilinear isometries on function algebras, Linear Multilinear Algebra 63 (2015), no. 7, 1448-1457.

[8] M. Jerison, The space of bounded maps into a Banach space, Annals of Mathematics 52 (1950) 309-327.

[9] A. Moreno and A. Rodríguez, A bilinear version of Holsztyński's theorem on isometries of C(X)-spaces, Studia Math. 166 (2005) 83-91.

[10] A. Rodríguez, Absolute valued algebras and absolute-valuable Banach spaces, Advanced courses of mathematical analysis I: Proc. First Intern. School, Cádiz, Spain, 2002, World Scientific Publ., 2004, 99-155.

[11] T. Tonev and R. Yates, Norm-linear and norm-additive operators between uniform algebras, J. Math. Anal. Appl. 357 (2009) 45-53.

Department of Mathematics, K.N. Toosi University of Technology, Tehran, 16315-1618, Iran

E-mail address: m.hosseini@kntu.ac.ir

Departamento de Matemáticas, Universitat Jaume i, Campus Riu Sec, 8029 AP, Castellón, Spain

E-mail address: font@mat.uji.es 